\title{
TIDAL DWARFS IN THE M81 GROUP: THE SECOND GENERATION?^
}

\author{
L. MAKAROVA ${ }^{1}$, E. GREBEL ${ }^{2}$, I. KARACHENTSEV ${ }^{3}$, A. DOLPHIN ${ }^{4}$, \\ V. KARACHENTSEVA ${ }^{5}$, M. SHARINA ${ }^{6}$, D. GEISLER ${ }^{7}$, P. GUHATHAKURTA ${ }^{8}$, \\ P. HODGE $^{9}$, A. SARAJEDINI ${ }^{10}$ and P. SEITZER ${ }^{11}$ \\ ${ }^{1}$ Special Astrophysical Observatory, Russia; E-mail: lidia@sao.ru \\ ${ }^{2}$ Max-Planck Institute for Astronomy, Heidelberg, Germany \\ ${ }^{3}$ Special Astrophysical Observatory, Russia \\ ${ }^{4}$ Kitt Peak National Observatory, NOAO, USA \\ ${ }^{5}$ Astronomical Observatory of Kiev University, Kiev, Ukraine \\ ${ }^{6}$ Special Astrophysical Observatory, Russia \\ ${ }^{7}$ Universidad de Concepción, Chile \\ ${ }^{8}$ UCO/Lick Observatory, USA \\ ${ }^{9}$ University of Washington, USA \\ ${ }^{10}$ University of Florida, USA \\ ${ }^{11}$ University of Michigan, USA
}

\begin{abstract}
We present a quantitative star formation history derivation of the four suspected tidal dwarf galaxies in the M 81 group: Holmberg IX, BK3N, Arp-loop (A0952+69) and Garland using HST/WFPC2 images of these galaxies. We construct a library of synthetic Colour-Magnitude Diagrams (CMDs) based on theoretical isochrones and data-derived determinations of photometric errors. These synthetic CMDs were combined linearly and $\chi^{2}$-compared to observed photometry. All the galaxies show continuous star formation between about 20 and $200 \mathrm{Myr}$ ago with star formation rates between $7.5 \cdot 10^{-3} \mathrm{M}_{\odot} / \mathrm{yr}$ and $7.6 \cdot 10^{-4} \mathrm{M}_{\odot} / \mathrm{yr}$. The metallicity of the detected stars is spanning rather a wide range, being lower than solar abundance. We suppose, that all the galaxies were formed out of material from metal-poor outer part of the giant spiral galaxy M81 after tidal interaction about $200 \mathrm{Myr}$ ago. However, this suggestion requires significantly more deep color-magnitude diagrams to be sure with the scenario of the galaxy evolution.
\end{abstract}

Keywords: dwarf galaxies, photometry, stellar content, M81 group

\section{Introduction}

Galaxy interactions are still common at the present epoch and can have a significant impact on appearance and evolution of galaxy groups and clusters. Evidence for dramatic interactions involving an entire galaxy group can be seen in the nearby M 81 group, whose dominant members M 81, M 82, NGC 3077, and NGC 2976 are embedded in a huge $\mathrm{HI}$ cloud with extended tidal H I bridges between the

^ Based on observations with the NASA/ESA Hubble Space Telescope, obtained at the Space Telescope Science Institute, which is operated by the Association of Universities for Research in Astronomy, Inc., under NASA contract NAS 5-26555. These observations are associated with proposal ID GO-8192.

Astrophysics and Space Science 285: 107-111, 2003.

(C) 2003 Kluwer Academic Publishers. Printed in the Netherlands. 
components. This strong interaction may be a reason for the formation of tidal dwarf galaxies from a condensed interstellar medium that was stripped from the main body of one of the galaxies in the interacting system.

In the framework of our Hubble Space Telescope (HST) snapshot survey of nearby dwarf galaxy candidates we have imaged 25 galaxies in the M 81 group. Photometric distances of these galaxies were obtained using the tip of red giant branch (TRGB), a well-established distance indicator. Here we present an analysis of data on four dwarf irregular galaxies located in the tidal $\mathrm{H}$ I features of the M 81 group (Holmberg IX, BK3N, Arp-loop and Garland).

\section{Observations, Data Reduction and CM Diagrams}

The galaxies were observed with the Hubble Space Telescope during 2000 - 2001 years with the Wide Field and Planetary Camera (WFPC2) as a part of the Dwarf Galaxy Snapshot Survey. For each target, two exposures of $600 \mathrm{sec}$ each were obtained, one in the F606W filter and one in the F814W filter. Stellar photometry of the galaxies was carried out by A.E. Dolphin with his HSTphot program (Dolphin, 2000).

Color-magnitude (V-I,I) diagrams of the four galaxies under consideration are presented in Figure 1. The magnitudes were not corrected for Galactic extinction. Isochrones of appropriate metallicities and ages from Girardi et al. (2000) are shown in the CM diagrams. The M 81 Cepheid distance modulus ( $\mu_{0}=27.80 \mathrm{mag}$, Freedman et al., 1994) was taken for the three galaxies Holmberg IX, BK3N, and Arp-loop. For Garland we used the distance modulus obtained from the tip of the red giant branch (TRGB) of the NGC 3077 halo population(Karachentsev et al., 2002). There are no clear signs of the red giant branch in the CMD of Holmberg IX. The resolved stellar populations are mainly blue and red supergiant stars.

\section{The Method}

We used the starFISH package by Harris and Zaritsky (2001) for the star formation history analysis. This package is intended to determine the best-fit star formation history $(\mathrm{SFH})$ of a mixed stellar population. Our photometry of the stars in the dwarf galaxies contains only stars sufficiently luminous to be detectable in 10-min exposures with HST/WFPC2. The number of stars in the CMDs is low (about 250 in Arp-loop, for example). These factors make the calculations especially difficult, and uncertainties in the star formation rate (SFR) estimation are rather large. Nevertheless, most of the galaxies have reduced goodness-of-fit $\chi^{2}<10$. These are quite good results according to J. Harris (starFISH User's Guide). 

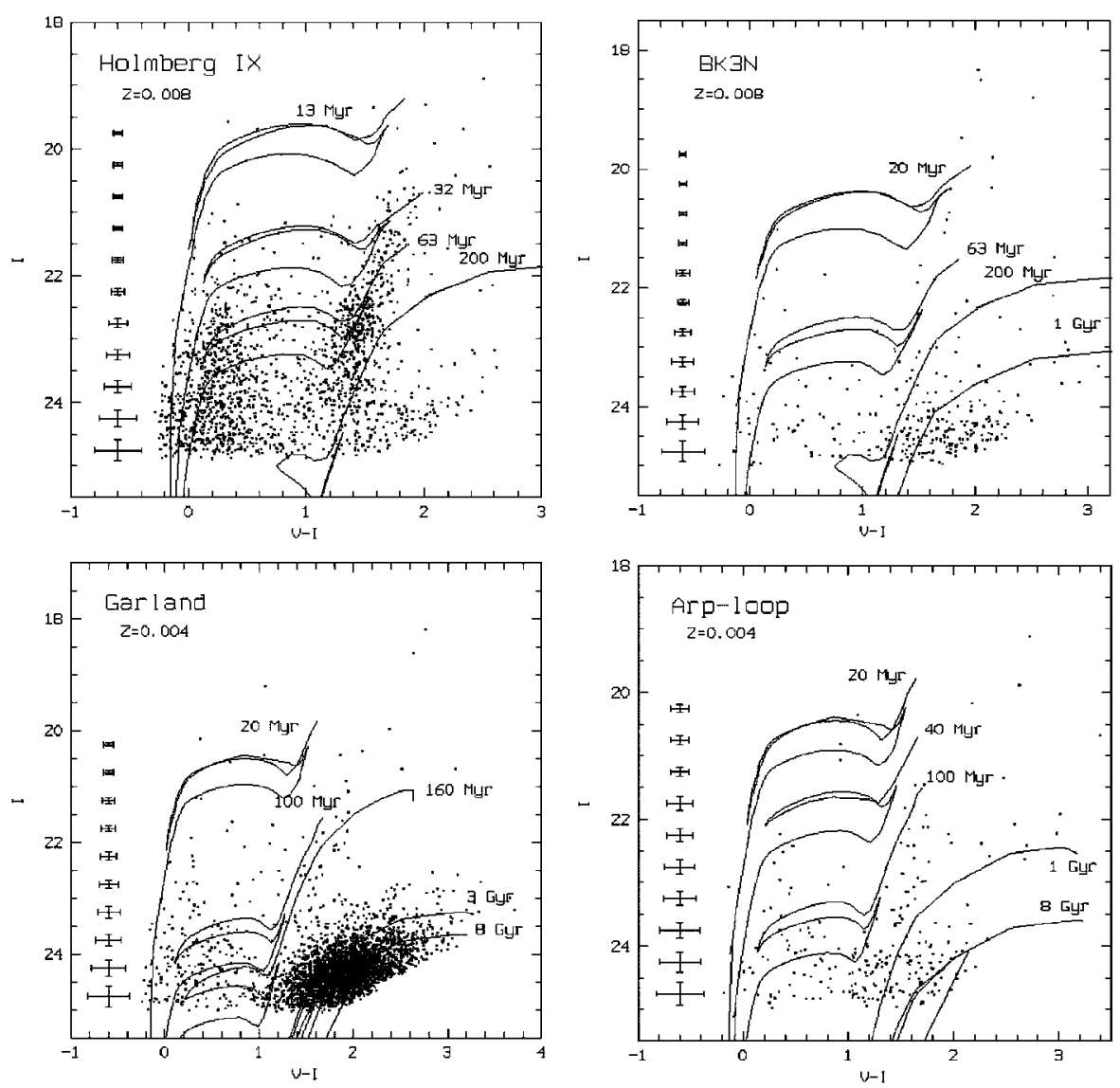

Figure 1. I,V-I color-magnitude diagrams of the dwarf irregular galaxies. The error bars on the left side of each panel show $1 \sigma$ distribution of the photometric errors. Isochrones by Girardi et al. (2000) are overplotted in the CMDs.

\section{Results and Discussion}

Table I summarizes the results of the synthetic CMD modelling of our HST/WFPC2 data for the suspected tidal dwarfs.

The photometric limits of our images do not allow us to clarify the evolutionary status of the four dwarf irregular galaxies with good accuracy. However, a comparison of the star formation ages in the galaxies derived via synthetic CMD modelling helps us to make some suggestions. As can be seen from the Table, Holmberg IX, BK3N, and Arp-loop (and probably Garland as well) experienced an episode of enhanced star formation between roughly 20 and 200 Myr ago. The star formation process may have been activated by the tidal interaction between M81, M82, and NGC3077, which is believed to have occurred 220 Myr ago (M81M82) and 280 Myr ago (M81-NGC3077) according to numerical simulations by Yun (1999). 
TABLE I

Star formation history of the tidal dwarfs

\begin{tabular}{ccccllc}
\hline Name & $\begin{array}{c}E(V-I) \\
{[\mathrm{mag}]}\end{array}$ & $\begin{array}{c}A_{I} \\
{[\mathrm{mag}]}\end{array}$ & $\begin{array}{l}\mu_{0} \\
{[\mathrm{mag}]}\end{array}$ & Star formation age & $\begin{array}{l}\text { mean SFR } \\
{\left[\mathrm{M}_{\odot} \mathrm{yr}^{-1}\right]}\end{array}$ & $\begin{array}{c}{[\mathrm{Fe} / \mathrm{H}]} \\
{[\mathrm{dex}]}\end{array}$ \\
\hline Holmberg IX & 0.109 & 0.154 & 27.80 & $6-200 \mathrm{Myr}$ & $7.5_{-2.2}^{+2.6} \cdot 10^{-3}$ & $-0.7 \div-0.4$ \\
\hline BK3N & 0.110 & 0.155 & 27.80 & $8-200 \mathrm{Myr}$ & $3.8_{-1.7}^{+8.9} \cdot 10^{-4}$ & $-0.7 \div 0.0$ \\
& & & & $500 \mathrm{Myr}-2 \mathrm{Gyr}$ & $0.8_{-0.5}^{+13.4} \cdot 10^{-4}$ & -0.7 \\
& & & & $8-12 \mathrm{Gyr}$ & $2.0_{-1.4}^{+2.9} \cdot 10^{-4}$ & -0.7 \\
\hline \multirow{2}{*}{ Arp-loop } & 0.117 & 0.164 & 27.80 & $40 \mathrm{Myr}-160 \mathrm{Myr}$ & $3.6_{-2.1}^{+9.7} \cdot 10^{-4}$ & $-1.3 \div-0.4$ \\
& & & & $500 \mathrm{Myr}-1 \mathrm{Gyr}$ & $2.6_{-1.9}^{+13.9} \cdot 10^{-4}$ & $-1.3 \div-0.4$ \\
& & & & $5-8 \mathrm{Gyr}$ & $2.2_{-1.6}^{+5.2} \cdot 10^{-4}$ & $-1.3 \div-0.7$ \\
\hline \multirow{2}{*}{ Garland } & 0.092 & 0.130 & 27.89 & $16-200 \mathrm{Myr}:$ & & $-0.7 \div 0.0$ \\
& & & & $600 \mathrm{Myr}-1 \mathrm{Gyr}:$ & & $-0.7 \div 0.0$ \\
& & & & $8-10 \mathrm{Gyr}:$ & & $-0.7 \div 0.0$ \\
\hline
\end{tabular}

There are also intermediate age stars in Arp-loop and BK3N (and probably in Garland, too). These stars may indicate that these two (three) galaxies existed already prior to the tidal interaction, or contain older stellar material torn out of the massive galaxies during the interaction. Finally, some star formation may have occurred in Arp-loop and BK3N $\geq 8$ Gyr ago, but these older stars have magnitudes close to our detection limit, which makes their interpretation difficult. While the young populations show a clear spatial coincidence and concentration toward the centers of the dwarf galaxies, this is less pronounced for the older populations. The proposed old red giant branch population may in fact belong to the extended old population of M81 and could be seen in projection along the line of sight to the dwarfs. In the case of Holmberg IX no significant indications of star formation longer ago than $\sim 200$ Myr ago could be detected.

Judging from the results of the synthetic CMD modelling, the metallicity of the detected stars shows a spread of up to 1 dex and is relatively high (up to -0.4 dex in $[\mathrm{Fe} / \mathrm{H}]$ for most star formation episodes). While the caveats of deriving photometric metallicities of young populations via isochrones should be kept in mind, these relatively high values (for low-mass dwarf galaxies) may support a tidal origin, although they remain below the abundances measured in M81 H II regions.

Taking into account the similarity of the star formation histories of the four dwarfs, we can suppose, that all the galaxies were formed out of material from metal-poor outer part of the giant spiral galaxy M81 after tidal interaction about 
200 Myr ago. However, this suggestion requires significantly more deep colormagnitude diagrams to be sure with the scenario of the galaxy evolution.

\section{Acknowledgements}

LM thanks the organization for giving her the opportunity to present this work at a pleasant and interesting conference. LM acknowledges partial funding from the conveners of the 'Galaxy evolution' workshop through project ref. PESO/PRO/ 15130/1999 from FCT/ Portugal and from EAS which allowed to present this work at the JENAM2002 meeting. Travel of LM to JENAM-2002 was also partially supported through RFBR Grant 02-02-27356. The work of LM was supported by INTAS grant YSF 2001/1-0129 and by the Max-Planck Institute for Astronomy, Heidelberg. LM, IK and MS acknowledge support through the RFBR Grant 0116001.

\section{References}

Dolphin, A.E.: 2000, PASP 112, 1383.

Freedman, W.L., Hughes, Sh.M., Madore, B.F. et al.: 1994, ApJ 427, 628.

Girardi, L., Bressan, A., Bertelli, G. and Chiosi, C.: 2000, A\&AS 141, 371.

Harris, J. and Zaritsky, D.: 2001, ApJ Suppl. 136, 25.

Karachentsev, I.D., Dolphin, A.E., Geisler, D., Grebel, E.K., Guhathakurta, P., Hodge, P.W., Karachentseva, V.E., Sarajedini, A., Seitzer, P. and Sharina, M.E.: 2002, A\&A 383, 125.

Yun, M.S.: 1999, IAU Symp. 186, 81. 
